\title{
Apparent motion: Evidence of the influence of shape, slant, and size on the correspondence process
}

\author{
ARIEN MACK, LAURIE KLEIN, JAMES HILL, and DONNA PALUMBO \\ New School for Social Research, New York, New York
}

\begin{abstract}
Four stimulus elements configured as a notional diamond were flashed in pairs to elicit apparent motion. When the elements were identical ( $4 \mathrm{Zs}$ ), the direction of apparent motion was ambiguous. When the elements were pairs of different letters ( $C \mathrm{~s}$ and $O \mathrm{~s}, E \mathrm{~s}$ and $Z \mathrm{~s}$ ), letters of different sizes ( $Z s$ and $z \mathrm{~s}$ ), or oppositely oblique lines, the direction of apparent motion tended to be between identical elements. This was true, however, only for an initial, brief observation period. Subsequently, the direction of apparent motion tended to be determined by the direction of motion perceived at first, regardless of the character of the elements. This quickly established directional set (within $10 \mathrm{sec}$ ) largely swamped any tendency to resolve correspondence in terms of a feature of the stimulus. It appears to be based on spatial rather than retinal or egocentric coordinates.
\end{abstract}

The phenomenon of apparent motion (AM) has been studied intensively in the 75 years since it was first richly described by Wertheimer (1912). Nevertheless, uncertainty and disagreement about some of its characteristics persist. For example, it is still not clear whether shape, orientation, or size plays a role in the matching process responsible for the pairing of successively appearing elements-a process referred to as correspondence (Ullman, 1979). A brief review of some of the relevant literature will make this clear.

Several investigators have reported that shape influences correspondence (Berbaum, Lenel, \& Rosenbaum, 1981; Kolers \& Pomerantz, 1971; Orlansky, 1940). In contrast, at least one other investigator has reported that shape plays no role (Navon, 1976). Several investigators have reported that orientation influences correspondence (Green, 1986; Ullman, 1980), while at least one study has reported the opposite (Burt \& Sperling, 1981). Both Ullman (1980) and Navon (1976) report that size is a correspondence token, whereas Burt and Sperling (1981) have found that it is not. Finally, there is some evidence that low spatial frequencies serve as correspondence tokens (Green, 1986; Ramachandran, Ginsburg, \& Anstis, 1983; Watson, 1986), but investigators who have found this evidence disagree about the influence of high spatial frequencies.

There are at least two factors which might account for these discrepancies by masking any tendency toward feature correspondence in ambiguous AM displays. One is a directional set, which our preliminary study suggested develops rapidly when subjects observe ambiguous motion displays, and which we studied in the present experi-

This research was supported by NSF Grant BNS 8310811. Correspondence may be addressed to Arien Mack, Psychology Department, New School for Social Research, 65 Fifth Avenue, New York, NY 10003. ments. ${ }^{1}$ The other is a strong vertical anisotropy that occurs in AM displays composed of sequentially flashed pairs of elements placed at the corners of a notional square (e.g., see Navon, 1976). Unlike the set effect, this effect is well documented (Gengerelli, 1948; Hoeth, 1968; Kruse, Stadler, \& Wehner, 1986; Ramachandran \& Anstis, 1983), and it has been attributed to the fact that the vertically aligned elements stimulate the same cortical hemisphere, whereas the horizontal elements stimulate opposite hemispheres (Gengerelli, 1948). Since the effect is reversed when the head is tilted $90^{\circ}$, this explanation appears to be correct.

Experiment $1 \mathrm{~A}$ looks at the influence of the identity of shape and line orientation in the correspondence process, under conditions that eliminate the problems of vertical anisotropy and directional set. The anisotropy was eliminated with the sequential presentation of pairs of elements at opposite apices of a notional diamond, thereby ruling out differential hemispheric stimulation. The possibility of a directional set was minimized with the use of a single 10-sec observation period, which was long enough for naive observers to get a clear sense of the direction of motion and short enough so that a switch in the direction of AM was unlikely. We chose stimulus elements and temporal parameters similar to those Navon used. In subsequent versions of this experiment-Experiments $1 B$ and $1 \mathrm{C}$-we investigated the set effect by presenting the observers with a second observation period.

\section{EXPERIMENT 1A}

\section{Method}

Subjects. Forty-two observers with normal or corrected-to-normal vision were recruited from the New School student population. None of the observers had previously participated in an AM study.

Apparatus and Procedure. The stimuli were displayed on a screen linked to a Commodore 64 microprocessor. The background 


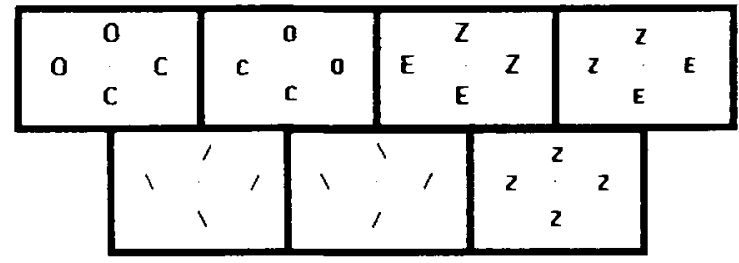

Figure 1. Schematic drawings of the various displays used in Experiments $1 \mathrm{~A}, 1 \mathrm{~B}$, and $1 \mathrm{C}$.

luminance measured by an Osabi Pentax $1^{\circ}$ spot meter was $2.806 \mathrm{~cd} / \mathrm{m}^{2}$. The luminance of the stimuli was $5.616 \mathrm{~cd} / \mathrm{m}^{2}$. There were seven different displays, six of which consisted of different orderings of pairs of the letters $C$ and $O$, of the letters $Z$ and $E$, and of $45^{\circ}$ oppositely oblique lines. ${ }^{2}$ The seventh display, which served as a control, consisted of four Zs. These displays, pictured in Figure 1, were viewed from a distance of $45 \mathrm{~cm}$. Each of the seven displays had a larger and smaller version, which, for the sake of brevity, will be referred to as the large or the small display. ${ }^{3}$ Five of the displays were composed of letters, and two of lines.

The dimensions of the large displays were as follows: Each letter subtended $1.2^{\circ}$ of visual angle vertically and $.95^{\circ}$ horizontally. The largest vertical and horizontal distance separating letters was $6.87^{\circ}$, and the separation between the midpoints of adjacent letters was $4.0^{\circ}$. The large oblique lines subtended an angle of $1.95^{\circ}$, and the largest vertical and horizontal separation between lines was $7.1 \% \mathrm{~cm}$. The separation between midpoints of adjacent lines was $3.9^{\circ}$. The dimensions of the small letter displays were as follows: Each letter subtended an angle of $.46^{\circ}$ horizontally and vertically. The largest vertical and horizontal distances between letters was $1.8^{\circ}$. Each small line subtended an angle of $.69^{\circ}$, and the largest vertical and horizontal separation between them was $1.8^{\circ}$. The separation between midpoints of adjacent lines was $.92^{\circ}$. There was a small fixation dot at the center of each display, and all displays were viewed in normal room light by observers whose heads were stabilized with a chin rest.

Each pair of stimulus elements was presented for $150 \mathrm{msec}$, and the ISI was either 32 or $16 \mathrm{msec}^{4}$ In every instance, different elements that were located directly opposite each other appeared together, for example,

$$
Z \cdot e, \text { followed by } \stackrel{E}{\dot{Z}}
$$

AM based on correspondence of shape or slant was evidenced by the perception of motion between identical elements-diagonally down to the right and up to the left in the illustrated case. Approximately $1 \mathrm{sec}$ before the display appeared, the fixation point became visible. The observers were alerted to the fact that a set of elements would be displayed on the screen for a brief period of time $(10 \mathrm{sec})$. They were asked to note whether the elements appeared to move, and, if so, to note the direction of motion so that they could describe it after the display disappeared. They were also asked to report whether the direction of motion appeared to change.

In order to give the observers a sense of the task at hand, an unambiguous AM configuration consisting of three sequentially presented, equally spaced, and horizontally aligned points was shown prior to the actual testing. Each subject viewed only one test display. Six subjects viewed each of the different arrays; 3 of these subjects viewed the large, and 3 the small displays.

\section{Results}

Since there were no differences between the responses to the large and small versions of the display, the results were combined. They are presented in Table 1. The results with the control display confirm its ambiguity. On trials in which feature correspondence was possible, the ratio of correspondence to noncorrespondence reports was 4.4 to 1 . An analysis of these results yielded a $\chi^{2}=28.5$, with $p<.001$. Thus, when composed of identical elements, this array gives rise to ambiguous AM; but it is significantly biased toward shape and slant correspondence when that possibility is instead present.

\section{EXPERIMENT 1B}

Experiment 1B was designed to confirm the initial results and examine the possibility of a directional set. To this end, a second trial was included, containing a different display, in which feature-based correspondence was opposite in direction to that in the array seen first. The time between the first trial and the second trial was a few minutes, but it seemed clear from our own informal observations that once a directional set had developed, it endured.

\section{Method}

Subjects. Forty-eight observers with normal or corrected-tonormal vision were tested. No observers had previously participated in an AM study.

Apparatus and Procedure. These were essentially the same as in Experiment 1A. The one difference was that in Experiment 1B, each observer viewed two different displays, each one for $10 \mathrm{sec}$. The set of elements from which the displays were generated remained the same. The elements of the two arrays and the direction of motion that signified correspondence based on shape or slant always differed in the two displays. The sizes of the elements in the two different arrays viewed by each observer were not necessarily different. The instructions to the observers were identical to those in the earlier version. Thirty-six subjects viewed two arrays that allowed for correspondence based on shape or slant. The remaining 12 subjects viewed the control display consisting of $\mathrm{Zs}$, fol-

Table 1

Percentages of Responses, Experiment 1A

\begin{tabular}{cccccc}
\hline Group & Corr & Noncorr & cw/ccw & $\backslash$ & $/$ \\
\cline { 1 - 2 } $\begin{array}{l}\text { Experimental }(N=36) \\
\text { Control }(N=6)\end{array}$ & $75 \%$ & $17 \%$ & $8 \%$ & & \\
\hline
\end{tabular}

Note-Slashes above columns represent right or left apparent motion. $\mathrm{cw}=$ clockwise, $\mathrm{cww}=$ counterclockwise, Corr $=$ correspondence, Noncorr $=$ noncorrespondence. 
Table 2

Percentages of Responses, Experiment 1B

\begin{tabular}{|c|c|c|c|c|c|}
\hline \multirow[b]{2}{*}{ Group } & \multirow[b]{2}{*}{ Corr } & \multirow[b]{2}{*}{ Noncort } & \multicolumn{3}{|c|}{ Direction of Apparent Motion } \\
\hline & & & $\mathrm{cw} / \mathrm{cww}$ & 1 & 1 \\
\hline \multicolumn{6}{|c|}{ Trial 1} \\
\hline $\begin{array}{l}\text { Experimental }(N=36) \\
\text { Control }(N=12)\end{array}$ & $78 \%$ & $11 \%$ & $\begin{array}{l}11 \% \\
16 \%\end{array}$ & $50 \%$ & $33 \%$ \\
\hline \multicolumn{6}{|c|}{ Trial 2} \\
\hline $\begin{array}{l}\text { Experimental }(N=36) \\
\text { Control }(N=12)\end{array}$ & $\begin{array}{l}42 \% \\
50 \%\end{array}$ & $\begin{array}{l}50 \% \\
42 \%\end{array}$ & $\begin{array}{l}5 \% \\
8 \%\end{array}$ & & \\
\hline
\end{tabular}

Note-Slashes above columns represent right or left apparent motion. $\mathrm{cw}=$ clockwise, $\mathrm{ccw}=$ counterclockwise, Corr $=$ correspondence, Noncorr $=$ noncorrespondence.

lowed by one of the displays that afforded the possibility of feature correspondence. The results from these subjects were meant to confirm those from the control subjects in the earlier version, as well as to indicate whether viewing the control display also established a directional set.

\section{Results}

The results are summarized in Table 2 . The first-trial results with the control display are consistent with the comparable results from Experiment 1A. The results from the first trial with displays that afforded the possibility of feature correspondence are also consistent with the previous results. This time, the ratio of correspondence to noncorrespondence reports was 7 to 1 , and an analysis of these results yielded a $\chi^{2}=13.82$, with $p<.001$, again indicating a significant tendency toward correspondence based on shape or slant.

The results from the second trial fall into a quite different pattern. There is now no evidence of shape or slant correspondence. On the contrary, these displays now appear to be as ambiguous as the control display composed of four identical elements. However, if we parse these results in terms of whether the direction of motion perceived in the first trial persists into the second, we find that $71 \%$ saw the same direction of motion. (One subject failed to see motion in the second display and so was dropped from this calculation.) Of these $71 \%, 81 \%$ saw motion opposite in direction to that consistent with shape or slant correspondence. ${ }^{5}$ An analysis of these results in terms of the significance of changes (Siegel, 1956, p. 63) indicates a significant tendency to preserve direction from the first to the second trial: $\chi^{2}=6.8, p<.005$. We take this as evidence of the operation of a directional set, which seems likely to swamp a tendency to perceive the direction of AM in terms of shape or slant correspondenceat least given the set of stimulus elements used.

There is also no significant evidence of feature correspondence in the reports of the 12 observers who saw the control display first. There is, however, again a tendency to preserve the initially perceived direction of motion, although this tendency seems less pronounced. Fiftyeight percent of the subjects maintained this direction on the second trial. ${ }^{6}$ The final version of this experiment, Experiment $1 \mathrm{C}$, was designed to investigate whether the tendency toward shape or slant correspondence plays any role at all on the second encounter with an AM display. To answer this question, we had subjects view the control display after viewing one of the other displays. If feature correspondence does inhibit the effectiveness of a directional set, there should have been more cases of the perseveration of direction here than in Experiment $\mathbf{l B}$, in which subjects viewed arrays that allowed for feature correspondence on the second trial.

\section{EXPERIMENT 1C}

\section{Method}

Subjects. Twelve subjects with normal or corrected-to-normal vision were tested. None of the subjects had any prior experience in an AM study.

Procedure. The procedures were identical to those used earlier, except that the second trial involved exposure to the control display. All 12 different versions of the display were used, 6 of which were large and 6 small (these displays are described in the Method section of Experiment 1A).

\section{Results.}

The results from the first trial are presented in Table 3. The ratio of correspondence to noncorrespondence reports was 10 to $1\left(\chi^{2}=13.8, p<.001\right)$. Ninety-two percent persisted in seeing motion in the same direction on the second trial. Even though a greater percentage of the subjects maintained direction here than in Experiment 1B, this difference, though in the expected direction, is not significant $\left[\chi^{2}(1)=1.07\right]$. Thus the data do not allow the conclusion that a tendency to feature correspondence opposes the directional set once it has been established.

\section{EXPERIMENT 2A}

Both Navon (1976) and Ullman (1980) reported that size is likely to influence the resolution of the correspondence problem. In Experiments 2A and 2B, we assessed the relative strengths of shape (slant) and size similarity. We

Table 3

Percentages of Responses, Experiment 1C, Trial 1

\begin{tabular}{cccc}
\hline Group & Corr & Noncorr & $\mathrm{cw} / \mathrm{ccw}$ \\
\hline Experimental $(N=12)$ & $83 \%$ & $8.5 \%$ & $8.5 \%$
\end{tabular}

Note-cw $=$ clockwise apparent motion, $\mathrm{ccw}=$ counterclockwise, Corr $=$ correspondence, Noncorr $=$ noncorrespondence. 
wished to determine whether, when shape or slant conflicted with size, there was any clear tendency for correspondence to be resolved in terms of size identity when the perceived direction of AM was uninfluenced by a previously established directional set. Displays were created in which correspondence could be resolved in terms of either shape (slant) or size identity. If size correspondence has a higher valence than shape or slant, the direction of AM ought predominantly to be consistent with the matching of size.

A second trial was included to determine whether the influence of a directional set might be reduced by the possibility of resolving correspondence in terms of size identity.

\section{Method}

Subjects. Fifteen observers with normal or corrected-to-normal vision were tested. None had previous experience with AM studies.

Procedure. There were three different displays. The elements were the same as those used earlier (Cs and $O s, Z s$ and $E s$, and opposite oblique lines). The principal difference was in the size of the elements. Within each simultaneously presented pair of elements, one element was larger than the other, and the sizes of the elements switched as they were cycled. The large and small elements had the same dimensions as the comparable elements in Experiments 1A-C. The displays were arranged so that the paired elements were either different letters of different sizes or opposite oblique lines of different sizes. This meant that resolution of the correspondence problem always involved a choice between a size or shape (slant) match, for example

$$
Z \cdot e, \text { followed by } \stackrel{z}{\dot{E}}
$$

The largest vertical and horizontal separation between the elements was $6.6^{\circ}$ and the separation between midpoints of adjacent elements was $4.1^{\circ}$. The temporal parameters were identical to those in the previous experiments.

Each subject viewed two different arrays. The second display was always composed of elements that were different from those seen first, and they were configured so that a size identity match was opposite in direction to that in the first display. The first array was viewed for $10 \mathrm{sec}$. The second array was viewed for a maximum of $60 \mathrm{sec}$, or until the observer reported a switch in the direction of AM by depressing a key on the computer keyboard-which turned off an interval timer and enabled the recording of the duration of the observation period. This manipulation permitted the determination of whether, if there is a perseveration of direction from first to second trial, it is more or less likely to endure if it is consistent with size correspondence. Prior to the second trial, the observers were told about the possibility that the direction of the motion between elements might change, and that if it did, they were to depress a key on the computer, which would turn off the display. They were then to report the initial direction of the AM.

\section{Results}

Results from the first trial are reported in Table 4. Fewer observers reported AM in the direction of size cor-

Table 4

Percentages of Responses, Experiment 2A, Trial 1

\begin{tabular}{ccc}
\hline Group & $\begin{array}{c}\text { Size } \\
\text { Correspondence }\end{array}$ & $\begin{array}{c}\text { Shape/Slant } \\
\text { Correspondence }\end{array}$ \\
\hline Experimental $(N=15)$ & $40 \%$ & $60 \%$ \\
\hline
\end{tabular}

Table 5

Percentages of Responses, Experiment 2B, Trial 1

\begin{tabular}{|c|c|c|}
\hline Group & Size Correspondence & Noncorrespondence \\
\hline$\overline{\text { Experimental }(N=10)}$ & $90 \%$ & $10 \%$ \\
\hline
\end{tabular}

respondence. Size identity does not seem to have a privileged role in resolving correspondence. On the second trial, $93 \%$ reported motion in the same direction as in the first trial, thus showing a powerful directional set. The time taken to switch directions also provided no evidence that size is privileged over shape or slant. ${ }^{7}$

\section{EXPERIMENT 2B}

The influence of size identity unopposed by shape or slant identity was assessed in Experiment 2B.

\section{Method}

Subjects. Ten subjects with normal or corrected-to-normal vision were recruited from the New School student population. None had previously participated in a study of AM.

Procedure. Except for the fact that all four elements were identical in shape (Zs) and differed only in size, Experiments $2 \mathrm{~A}$ and $2 \mathrm{~B}$ were identical.

\section{Results}

The results from the first trial are reported in Table 5 . On the second trial, only 3 subjects initially perceived AM in the direction consistent with a size match. Eighty percent, however, initially reported motion in the same direction as in Trial 1, again providing evidence of a strong directional set. Two subjects reported motion in the opposite direction. ${ }^{8}$ There is no evidence that size correspondence is less likely to be swamped by a directional set.

\section{EXPERIMENT 3}

This experiment was designed to investigate whether the directional set is based on retinal (egocentric) or postretinal, spatial directions. Since egocentric and spatial directions are identical when the arrays are viewed with an upright head, we asked observers to view the second display with their heads inverted. If the observer reports the same spatial direction of motion with his or her head inverted, it is likely that the set is based on spatial direction, whereas if the direction reported on the second trial is opposite that reported on the first, it is likely to be retinal.

\section{Method}

Subjects. Twenty-four subjects who had not been in any of the previous experiments participated.

Procedure. The first array consisted of successively presented $E s$ and Zs. The array on the second trial consisted of pairs of Zs. On the first trial, observers viewed the display in the normal upright position, whereas on the second trial, they viewed the display while lying stomach down on a table with their heads extended over its edge. The computer screen was placed beneath the table and was therefore viewed with the head upside down. Viewing distance was $45 \mathrm{~cm}$, and the central fixation mark was approximately at eye level in both conditions. Each trial lasted $10 \mathrm{sec}$. 


\section{Results}

Fifty-four percent of the subjects reported AM based on shape identity in Trial 1 . There is no clear explanation for why fewer of these subjects resolved correspondence in terms of feature identity than in the previous experiments with the same stimuli and temporal parameters. Perhaps the reason is that since in no case did we find all subjects resolving correspondence in terms of shape, slant, or size identity, we accidentally tested a subject group with an unrepresentatively small number of observers opting for feature matching. This seems likely, since in all our experiments, some of which are unreported because they differed in some small way from the experiments reported and because they added no new information, the ratio of correspondence to noncorrespondence reports consistently ranged between 4 and 5 to 1 . Seventynine percent of the subjects maintained the same spatial direction from the first to the second trial. (One subject failed to see motion on Trial 2. If this subject is eliminated from the calculation, then $83 \%$ maintained the same spatial direction.) These results strongly suggest that the set is based on postretinal directions at a level in the processing of information at or after the level at which head position is taken into account.

\section{DISCUSSION}

There are several conclusions that may be safely drawn from these combined results:

1. There is evidence for a significant tendency to resolve correspondence in an ambiguous AM display in terms of shape, slant, or size identity when it is first encountered, at least with the small set of stimuli used in these experiments. This conclusion is supported by the combined results from all the first trials, in which shape, slant, or size correspondence was a possibility. There were 118 such instances. Of these, 89 (75\%) were resolved in terms of shape, slant, or size correspondence, which is, of course, well above the $50 \%$ that would be expected by chance. (If we allow for the possibility that these displays might equally reasonably be expected to give rise to clockwise or counterclockwise circular motion, then the estimate of what might be expected by chance is, of course, reduced, making the correspondence results even more impressive.)

2. On the basis of the combined data, there is no reason to expect that the second encounter with an ambiguous AM display will be resolved in terms of shape, slant, or size correspondence if that is an option. Again, this conclusion is limited to the small numbers of stimuli tested. There were 58 instances in which this was a possibility. Of these instances, only 24 , or $41 \%$, were resolved in terms of feature correspondence.

3 . There is a strong probability that a directional set developed on the first encounter with an ambiguous AM display will persist and determine the perceived direction of AM on the next encounter. This is so even when the direction of AM might be determined by matching elements in successive frames in terms of shape, slant, or size. There were 109 instances in which a directional set could have influenced the direction of AM. Of these 109 instances, 78 , or $71 \%$, were consistent with the determination of the perceived direction of AM by a directional set. ${ }^{9}$

4. There is some evidence that feature correspondence may play a role in determining the direction of AM, after a directional set has been established. When a directional set is unopposed by the possibility of a resolution of the correspondence problem in terms of a feature of the stimulus (Experiments $1 \mathrm{C}$ and 3, where the control array was viewed on the second trial, and Experiment $2 \mathrm{~A}$, where the two principal paths of AM were equally consistent with matching on the basis of a feature of the stimulus elements), 44 of the 50 observations (88\%) are consistent with a directional set. However, when the second trial poses a choice between feature correspondence and a direction match, 32 of the 45 observations $(71 \%)$ are consistent with a directional set (Experiments $1 B$ and 2B). This difference in frequency is significant $\left[\chi^{2}(1)=2.43\right.$, $p>.05$, one-tailed], therefore suggesting that when feature correspondence is possible, it continues to exert some influence on AM. This conclusion concerning correspondence is, of course, restricted to the set of stimuli tested.

5. Finally, the directional set appears to be spatial rather than retinal, and it is consistent with the initially perceived direction of AM. Were correspondence based on retinal matches, this would suggest that a later stage of processing was interrupting an earlier stage. It should be noted, however, that with the exception of proximity (Tarr \& Pinker, 1985; Ullman, 1979), there is no clear evidence concerning whether any of the features that serve as correspondence tokens, such as orientation, size, shape, or even spatial frequency, are predicated on retinal or postretinal representations.

We do not know why there is a directional set or why it tends to influence the perceived path of AM strongly. Any attribution of the set to an astigmatism is ruled out by the results of Experiment $2 \mathrm{C}$. Were the directional set a function of an astigmatism, it should have reversed when the subjects viewed the displays with their heads inverted. It remains to be seen, of course, whether this kind of directional set has an equivalent influence over other correspondence tokens-such as spatial frequency, for example. The visual system seems to be operating in these instances as if the identity of the elements is irrelevant, and this outcome is compatible with the view that feature analysis is slower than the detection of location and motion (Navon, 1976). This view, however, is incompatible with the firsttrial results, which demonstrate a significant tendency to resolve the correspondence problem in terms of the identity of the elements. If motion and/or location analysis were faster than or preceded feature analysis, the perceived direction of AM ought to be random on the first trial. In fact, the first-trial results support the opposite 
view; that the analysis of features precedes or occurs in parallel with the analysis of motion or detection of location.

Evidence that a directional set plays an important role in AM might account for some of the discrepancies in earlier results. When evidence of shape, size, or slant correspondence has not been obtained, it is possible that a directional set has been operative. Whether or not this is so, however, these findings document a heretofore largely unexamined characteristic of AM that needs to be considered in future research.

\section{REFERENCES}

ANSTIS, S. M., Ramachandran, V. S. (1987). Visual inertia in apparent motion. Vision Research, 27, 755-764.

Berbaum, K., Lenel, J. C., \& Rosenbaum, M. (1981). Dimensions of figural identity in apparent motion. Journal of Experimental Psychology: Human Perception \& Performance, 7, 1312-1317.

Burt, P., \& SPERling, G. (1981). Time, distance and feature tradeoffs in visual apparent motion. Psychological Review, 88, 171-195.

GENGERELL, J. A. (1948). Apparent motion in relation to homonymous and heteronymous stimulation of the cerebral hernispheres. Journal of Experimental Psychology, 38, 592-599.

GREEN, M. (1986). What determines correspondence strength in apparent motion? Vision Research, 26, 596-607.

HOETH, F. (1968). Bevorzugte Richtungen bei stroboskopischen Alternativebewegungen. Psychologische Beitrage, 10, 494-527.

Kolers, P. A., \& Pomerantz, J. R. (1971). Figural change in apparent motion. Journal of Experimental Psychology, 87, 99-108.

Kruse, P., Stradler, M., \& Wehner, T. (1986). Direction and frequency specific processing in the perception of long-range apparent movement. Vision Research, 26, 327-335.

NAvoN, D. (1976). Irrelevance of figural identity for resolving ambiguities in apparent motion. Joumal of Experimental Psychology: Human Perception \& Performance, 2, 130-138.

OrLaNSKY, J. (1940). The effect of similarity and difference in form on apparent visual movement. Archives of Psychology, 246.

Ramachandran, V. S, \& ANSTis, S. M. (1983). Perceptual organization in moving displays. Nature, 304, 829-831.

Ramachandran, V. S., Ginsburg, A., \& ANSTIS, S. M. (1983). Low spatial frequencies dominate apparent motion. Perception, 12, 457-461.

SIEGEL, S. (1956). Nonparametric statistics for the behavioral scientist. New York: McGraw-Hill.

TARR, M., \& PINKER, S. (1985, November). Nearest neighbors in apparent motion: Two or three dimensions? Paper presented at the meeting of the Psychonomic Society, Boston.

Ullman, S. (1979). The interpretation of visual motion. Cambridge, MA: MIT Press.

Ullman, S. (1980). The effect of similarity between line segments on the correspondence strengths in apparent motion. Perception, 9, 617-626.

Watson, A. B. (1986). Apparent motion occurs only between similar spatial frequencies. Vision Research, 26, 1727-1750.

WERTHEImER, M. (1912). Experimentelle Studien über das Sehen von Bewegung. Zeitschrift für Psychologie, 61, 161-265.

\section{NOTES}

1. While we were writing this paper, Anstis and Ramachandran (1987) published a report of a related phenomenon, which they describe as "visual inertia" in AM. Using a notional diamond-shaped array consisting of four identical dots in which the vertically aligned ones were alternated with the horizontal ones, they found clear evidence of priming: "If 2 priming dots were flashed beforehand in line with 2 parallel sides of the diamond, AM was seen along those sides" (p. 755). Although this effect differs from the one we observed, which occurred in the absence of priming and under conditions in which shape, slant, or size correspondence was possible, it too suggests the vulnerability of AM to a directional set.

2. Navon (1976), too, used $Z$ and $E$ pairs and pairs of oblique lines.

3. The dimensions of the elements in the small display approximated those used by both Navon (1976) and Ullman (1980). The overall (outer) dimensions of the large display approximated those used by Navon.

4. The temporal parameters were close to those reported by Navon (1976), who used the same 150 -msec presentation time and a 30-msec ISI. The temporal parameters were chosen for this reason. The variation in our ISI between 16 and $32 \mathrm{msec}$ was a performance characteristic of the microprocessor.

5. Since 1 subject failed to perceive motion on the second trial, that subject was eliminated from the calculations. Six of the remaining 35 subjects saw clockwise (cw) or counterclockwise (ccw) motion on either the first or second trial. If for these subjects the initial direction of motion in the second trial preserved the initial direction of motion in the first, that observation was scored as preserving direction. Four of these subjects fit this description. If we simply exclude all subjects who saw $\mathrm{cw}$ or ccw motion on one of the 2 trials, on the grounds that it is unclear whether or not directionality was preserved, then only 29 subjects remain in the pool. Of these, $21(72 \%)$ reported motion in the second trial that preserved the direction of motion in the first.

6. Of these 12 subjects, 3 saw $\mathrm{cw}$ or $\mathrm{ccw}$ motion on either the first or second trial. If these subjects are excluded from the calculation for the reason given in the previous note, it reduces the pool of subjects to 9 , which raises the percent maintaining direction to $78 \%$.

7. Twelve subjects reported a switch in the direction of AM in the second trial. Eight reported a switch from shape to slant correspondence, with the mean time for switching $17.5 \mathrm{sec}(S D=7.8 \mathrm{sec})$. Four subjects reported the opposite switch. The mean time for this switch was $11.2 \mathrm{sec}(S D=2.7 \mathrm{sec})$. Three subjects reported no switch in $60 \mathrm{sec}$. All of these subjects saw motion in the direction consistent with slant or shape correspondence.

8. Only 5 subjects reported a switch in the direction of AM. For 4 of these subjects, the switch was in the direction of a size match (mean switching time $=18.34 \mathrm{sec}, S D=4.9 \mathrm{sec}$ ). One subject switched in the opposite direction after $38.2 \mathrm{sec}$. These data may suggest a weak preference for a size match.

9. Ten observers, 4 of whom were the coauthors, looked at the AM displays intermittently over a period of several months. For half of these observers, the encounters with the AM displays were widely separated, yet for all 10 observers, the initial direction of AM persisted.

(Manuscript received March 21, 1988; revision accepted for publication January 24, 1989.) 